\title{
THE DRAG COEFFICIENT IN POWER-LAW NON-NEWTONIAN FLUID OVER MOVING SURFACE
}

\author{
Gabriella Bognár \\ Institute of Machine and Product Design, University of Miskolc \\ H-3515 Miskolc-Egyetemváros, Hungary \\ v.bognar.gabriella@uni-miskolc.hu
}

[Received: April 17, 2016]

\begin{abstract}
This paper deals with a solution to the boundary layer problem of a nonNewtonian power-law fluid flow along a moving flat surface. Two cases are investigated: one when the surface is moving in a fluid flow, the other when the surface is moving through an otherwise quiescent fluid. Applying similarity transformation to the system of the governing partial differential equations, the boundary value problem of one nonlinear ordinary differential equation on $[0, \infty)$ is derived. Numerical solutions obtained for the velocity components and the drag coefficient parameter depending on the velocity ratio and on the power-law exponent are exhibited.
\end{abstract}

Mathematical Subject Classification: 34B10, 34B15

Keywords: Non-Newtonian fluid, boundary layer, moving surface, similarity method, iterative transformation method, spectral method

\section{NomenClature}

$\begin{array}{lll}f & {[-]} & \text { similarity velocity } \\ g & {[-]} & \text { function } \\ h, h^{*} & {[-]} & \text { parameters } \\ K & {\left[\mathrm{~Pa} \mathrm{~s}^{n}\right]} & \text { consistency coefficient } \\ n & {[-]} & \text { power-law exponent } \\ u, v & {[-]} & \text { non-dimensional velocity components } \\ U_{\infty} & {[\mathrm{m} / \mathrm{s}]} & \text { fluid velocity } \\ U_{w} & {[\mathrm{~m} / \mathrm{s}]} & \text { wall velocity } \\ x, y & {[-]} & \text { non-dimensional variables }\end{array}$

\section{Greek symbols}

$\begin{array}{lll}\gamma, \sigma, \kappa, \mu & {[-]} & \text { parameters } \\ \Gamma & {[-]} & \text { function } \\ \eta & {[-]} & \text { similarity variable } \\ \rho & {\left[\mathrm{kg} / \mathrm{m}^{3}\right]} & \text { density of the fluid } \\ \psi & {[-]} & \text { non-dimensional stream function } \\ \tau_{y x} & {[\mathrm{~Pa}]} & \text { shear stress }\end{array}$




$\begin{array}{lll}\tau_{w} & {[\mathrm{~Pa}]} & \text { wall shear stress } \\ \lambda & {[-]} & \text { velocity ratio, } U_{\omega} / U_{\infty} \\ \lambda_{c} & {[-]} & \text { critical velocity ratio }\end{array}$

\section{INTRODUCTION}

In fluid dynamics, the drag force or force component in the direction of the flow velocity is proportional to the drag coefficient, to the density of the fluid, to the area of the object and the square of the relative speed between the object and the flow velocity. Blasius applied the similarity method to investigate the model arising for a laminar boundary layer of a Newtonian media [1. Fluids such as molten plastics, pulps, slurries and emulsions, which do not obey the Newtonian law of viscosity, are increasingly produced in the industry. The first analysis of the boundary layer approximations to non-Newtonian media with power-law viscosity was published by Schowalter [2] in 1960. The author derived the equations governing the fluid flow. Numerical solutions to the problem of a laminar flow of the non-Newtonian power-law model past a two-dimensional horizontal surface were presented by Acrivos at al. [3] When the geometry of the surface is simple the system of differential equations can be examined in details and fundamental information can be obtained about the flow behavior of a non-Newtonian fluid in motion (e.g., to predict the drag).

The production of sheeting material, which includes both metal and polymer sheets, arises in a number of industrial manufacturing processes. The fluid dynamics due to a continuous moving solid surface appears in aerodynamic extrusion of plastic sheets, cooling of a metallic plate in a cooling bath, the boundary layer along material handling conveyers, boundary layer along a liquid film in condensation processes, etc. Much theoretical work has been done on this problem since the pioneering papers by Sakiadis [4 and Tsou et al. 5], and extensive references can be found in the papers by Magyari and Keller [6], 7], Liao and Pop [8, and Nazar et al. [9].

Various types of non-Newtonian fluids are nano fluid, Casson fluid, viscoelastic fluid, couple stress fluid, micro polar fluid, power-law flow, etc. [10]. These include pseudo plastic, dilatant, blood, foodstuff, slurries, paints, cosmetics and toiletries. Heat transfer in boundary layer over a stretching sheet has important applications in extrusion of plastic sheets, polymer, spinning of fibers, cooling of elastic sheets, etc. (11], [12, [13]). The MHD boundary layer flow of heat and mass transfer problems about an stretching sheet have become important research topics in view of its significant applications in industrial manufacturing processes such as plasma studies, petroleum industries, magneto-hydrodynamics power generator, cooling of nuclear reactors, boundary layer control in aerodynamics, glass fiber production and paper production ([14, [15]).

Several numerical methods have been developed and introduced for the solution of these type of fluid mechanics' problems. It was shown in [16] that a non-iterative Töpfer-like transformation can be applied for the determination of the dimensionless wall gradient on a stationary flat surface. Due to the challenging problem of the solution of the boundary value problem to nonlinear differential equation(s) on infinite interval, it is beneficial to obtain the solution with different numerical simulations for 
comparison. The aims of the paper are to give numerical results on the drag coefficient in non-Newtonian media along moving flat surfaces for two cases and to introduce two numerical methods, an iterative transformation method and a spectral method, for the numerical evaluation.

\section{Mathematical Model}

Consider an incompressible uniform parallel flow of a non-Newtonian power-law fluid, with a constant velocity $U_{\infty}$ along an impermeable semi-infinite flat plate whose surface is moving with a constant velocity $U_{w}$ in the opposite direction to the main stream. The $x$-axis extends parallel to the plate, while the $y$-axis extends upwards, normal to it. Applying the necessary boundary layer approximations, the continuity and momentum equations are [17]:

$$
\begin{gathered}
\frac{\partial u}{\partial x}+\frac{\partial v}{\partial y}=0 \\
u \frac{\partial u}{\partial x}+v \frac{\partial u}{\partial y}=\frac{1}{\rho} \frac{\partial \tau_{y x}}{\partial y},
\end{gathered}
$$

where $u, v$ are the velocity components along $x$ and $y$ coordinates, respectively. The shear stress and the shear rate relation is assumed to be the power-law relation

$$
\tau_{y x}=K\left|\frac{\partial u}{\partial y}\right|^{n-1} \frac{\partial u}{\partial y},
$$

where $K$ stands for the consistency and $n$ is called the power-law index; that is $n<1$ for pseudoplastic, $n=1$ for Newtonian, and $n>1$ for dilatant fluids. Therefore, differential equation 2.2 is rewritten as

$$
u \frac{\partial u}{\partial x}+v \frac{\partial u}{\partial y}=\frac{\partial}{\partial y}\left(\mu_{c}\left|\frac{\partial u}{\partial y}\right|^{n-1} \frac{\partial u}{\partial y}\right)
$$

where $\mu_{c}=K / \rho$.

2.1. Fluid flow of velocity $U_{\infty}$. In the first case, the surface is placed in a fluid flow of velocity $U_{\infty}$. The wall is impermeable and no-slip boundary condition is supposed. For the investigated model, the boundary conditions are formulated as

$$
\left.u\right|_{y=0}=-U_{w},\left.\quad v\right|_{y=0}=0,\left.\quad u\right|_{y=+\infty}=U_{\infty}
$$

2.2. Flow in an otherwise quiescent fluid. In the second case, the ambient fluid velocity is zero and we suppose that the plate is moving at a constant velocity; therefore:

$$
\left.u\right|_{y=0}=-U_{w},\left.\quad v\right|_{y=0}=0,\left.\quad u\right|_{y=+\infty}=0 .
$$


2.3. Introduction of stream function. The continuity equation 2.1 is automatically satisfied by introducing a stream function $\psi$ as

$$
\begin{gathered}
u=\frac{\partial \psi}{\partial y}, \\
v=-\frac{\partial \psi}{\partial x} .
\end{gathered}
$$

The momentum equation can be transformed into an ordinary differential equation by the similarity transformations

$$
\begin{gathered}
\psi(x, y)=\mu_{c}^{\frac{1}{n+1}}\left(U_{\infty}\right)^{\frac{2 n-1}{n+1}} x^{\frac{1}{n+1}} f(\eta), \\
\eta=\mu_{c}^{-\frac{1}{n+1}}\left(U_{\infty}\right)^{\frac{2-n}{n+1}} y x^{-\frac{1}{n+1}},
\end{gathered}
$$

where $\eta$ is the similarity variable and $f(\eta)$ is the dimensionless stream function for the boundary value problem $(2.1),(2.3)$ and $(2.4)$. Equation 2.3 with the transformed boundary conditions can be written as

$$
\begin{gathered}
\left(\left|f^{\prime \prime}\right|^{n-1} f^{\prime \prime}\right)^{\prime}+\frac{1}{n+1} f f^{\prime \prime}=0, \\
f(0)=0, \quad f^{\prime}(0)=-\lambda, \quad f^{\prime}(\infty)=\lim _{\eta \rightarrow \infty} f^{\prime}(\eta)=1,
\end{gathered}
$$

where the prime denotes the differentiation with respect to the similarity variable $\eta$, and the velocity ratio parameter is $\lambda=U_{w} / U_{\infty}$. Equation 2.10 is called the generalized Blasius equation and the case $\lambda=0$ corresponds to the Blasius problem. It should be noted that for $\lambda>0$, the fluid and the plate move in opposite directions, while they move in the same directions if $\lambda<0$. Now, the dimensionless velocity components have the form:

$$
\begin{gathered}
u(x, y)=U_{\infty} f^{\prime}(\eta), \\
v(x, y)=\frac{U_{\infty}}{n+1} R e_{x}^{\frac{n}{n+1}}\left(\eta f^{\prime}(\eta)-f(\eta)\right),
\end{gathered}
$$

and

$$
\eta=R e_{x}^{\frac{1}{n+1}} y / x
$$

where

$$
R e_{x}=U_{\infty}^{2-n} x^{n} / \mu_{c}
$$

is the local Reynolds number.

For $U_{\infty}=0, \lambda$ is not defined. Here the momentum equation can be transformed into an ordinary differential equation by the similarity transformations

$$
\begin{gathered}
\psi(x, y)=\mu_{c}^{\frac{1}{n+1}}\left(U_{w}\right)^{\frac{2 n-1}{n+1}} x^{\frac{1}{n+1}} f(\eta), \\
\eta=\mu_{c}^{-\frac{1}{n+1}}\left(U_{w}\right)^{\frac{2-n}{n+1}} y x^{-\frac{1}{n+1}}
\end{gathered}
$$

for the boundary value problem 2.1), 2.3 and 2.4. The transformed form of equation (2.3) has the same form as 2.10), but the boundary conditions (2.5) are formed by

$$
f(0)=0, \quad f^{\prime}(0)=1, \quad f^{\prime}(\infty)=\lim _{\eta \rightarrow \infty} f^{\prime}(\eta)=0
$$


The dimensionless velocity components can be given as:

$$
u(x, y)=U_{w} f^{\prime}(\eta), \quad\left[v(x, y)=\frac{U_{w}}{n+1} R e_{x}^{\frac{n}{n+1}}\left(\eta f^{\prime}(\eta)-f(\eta)\right)\right.
$$

and

where

$$
\eta=R e_{x}^{\frac{1}{n+1}} y / x
$$

$$
R e_{x}=U_{w}^{2-n} x^{n} / \mu_{c}
$$

Since the pioneering work by Acrivos at al. [3], different approaches have been investigated for $f^{\prime \prime}(0)=\gamma$ in the case of non-Newtonian fluids. It has a physical meaning in drag force or force due to skin friction. It is a fluid dynamic resistive force, which is a consequence of the fluid and the pressure distribution on the surface of the object. The skin friction parameter $\gamma$ originates from the non-dimensional drag coefficient

$$
C_{D}=(n+1)^{\frac{1}{n+1}} R e^{\frac{-n}{n+1}}|\gamma|^{n-1} \gamma
$$

and it is involved in the wall shear stress

$$
\tau_{w}(x)=\left[\frac{\rho^{n} K U_{\infty}^{3 n}}{x^{n}}\right]^{\frac{1}{n+1}}|\gamma|^{n-1} \gamma
$$

for $U_{\infty} \neq 0$ and

$$
\tau_{w}(x)=\left[\frac{\rho^{n} K U_{w}^{3 n}}{x^{n}}\right]^{\frac{1}{n+1}}|\gamma|^{n-1} \gamma
$$

for $U_{\infty}=0$.

The boundary value problem 2.10, 2.11) is defined on a semi-infinite interval. For Newtonian fluids $(n=1)$, equation 2.10 is equal to the well-known Blasius equation:

$$
f^{\prime \prime \prime}+\frac{1}{2} f f^{\prime \prime}=0
$$

For non-Newtonian fluids on steady surfaces $(\lambda=0)$, the boundary value problem 2.10, 2.11 was investigated in [16]. A non-iterative Töpfer-like transformation was introduced for the determination of $\gamma$, when

$$
f(\eta)=\gamma^{(2 n-1) / 3} g\left(\gamma^{(2-n) / 3} \eta\right)
$$

and $g$ is the solution of the initial value problem

$$
\begin{gathered}
\left(\left|g^{\prime \prime}\right|^{n-1} g^{\prime \prime}\right)^{\prime}+\frac{1}{n+1} g g^{\prime \prime}=0, \\
g(0)=0, \quad g^{\prime}(0)=0, \quad g^{\prime \prime}(0)=1 .
\end{gathered}
$$

By analogy with the Blasius description of Newtonian fluid flows [1], here the goal is to study the similarity solutions and investigate the model arising in the study of a twodimensional laminar fluid flow with power-law viscosity. A Töpfer-like transformation is applied for the determination of $\gamma$. 


\section{Previous Results}

The existence and uniqueness of Blasius' boundary layer solution to $2.25,2.11$ with $\lambda=0$ was rigorously proved by Weyl [18. The properties of similarity solutions to the boundary layer problem on a moving surface $(\lambda \neq 0)$ for Newtonian fluids, have been examined by Hussaini and Lakin [19, Hussaini et al. [20]. It turned out that for a semi-infinite plate, the existence of solutions depends on the ratio of the plate surface velocity $U_{w}$ to the free stream velocity $U_{\infty}$. When $n=1, \lambda \leq 0$, the existence, uniqueness and analyticity of solution to 2.10, 2.11) were proved by Callegari and Friedman 21] using the Crocco variable formulation. If $\lambda>0$, Hussaini and Lakin [19] proved that there is a critical value $\lambda_{c}$ such that a solution exists to 2.25, 2.11 only if

$$
\lambda \leq \lambda_{c}
$$

(see [19]). Dual solutions exist for

$$
0<\lambda<\lambda_{c}
$$

The numerical value of $\lambda_{c}$ was found to be $0.3541 \ldots$ for $n=1$. The non-uniqueness and analyticity of the solution for

$$
\lambda \leq \lambda_{c}
$$

has been proved by Hussaini et al. 19, 20. For non-Newtonian fluids $(n \neq 1)$ with $\lambda=0$, the existence, uniqueness and some analytical results for problem 2.10, 2.11) were established when $0<n<1$ by Nachman and Callegari [22. The existence and uniqueness result for $n>1$ was considered by Benlahsen et al. [17] via Crocco variable transformation. For non-Newtonian fluids the numerical calculations also show that there is a critical value $\lambda_{c}$ for each $n$ such that solution exists only if

$$
\lambda \leq \lambda_{c}
$$

(see [23]). The variations of $f^{\prime \prime}(0)$ and $\lambda_{c}$ with $\lambda$ for different values of $n$ are given in 24].

In this paper an iterative transformation method is introduced for the determination of $\gamma$ involved in the drag coefficient and the calculation of the boundary layer thickness for different values $n$ and $\lambda$.

\section{Numerical SOLUtions}

For the determination of the solution to boundary value problems 2.10, 2.11) and 2.10 , 2.18 two different methods are applied. The problems are solved on truncated intervals instead of on $[0, \infty)$.

4.1. Iterative transformation method for the case $U_{\infty} \neq 0$. This section is devoted to the application of the scaling concept to numerical analysis of 2.10), 2.11. Solving this problem, proper boundary conditions are not available at infinity. 
In the case of $\lambda=0$ a non-iterative transformation method called Töpfer or Töpferlike method is be used for solving (2.10), 2.11) either for $n=1$ [7] or for $n \neq 1$ [24]. The figure of the velocity gradient parameter near the wall $f^{\prime \prime}(0)$ is exhibited in Figure 1 depending on $n$ [24].

Here an iterative transformation method is described. Non-iterative and iterative transformation methods for boundary value problems were introduced by Fazio [25]. The idea behind the present method is to consider the "partial" invariance of 2.10, 2.11 with respect to a scaling transformation in the sense that the differential equation and one of the boundary conditions at 0 are invariant, while the other two boundary conditions are not invariant. Therefore, the problem modified by introducing a numerical parameter $h$. Now, equation 2.10 is to be solved with boundary conditions

$$
\begin{gathered}
f^{\prime}(0)=-\lambda h, \\
f(0)=0, \quad f^{\prime}(\infty)=\lim _{\eta \rightarrow \infty} f^{\prime}(\eta)=1,
\end{gathered}
$$

where $h$ is involved, to ensure the invariance of the extended scaling group.

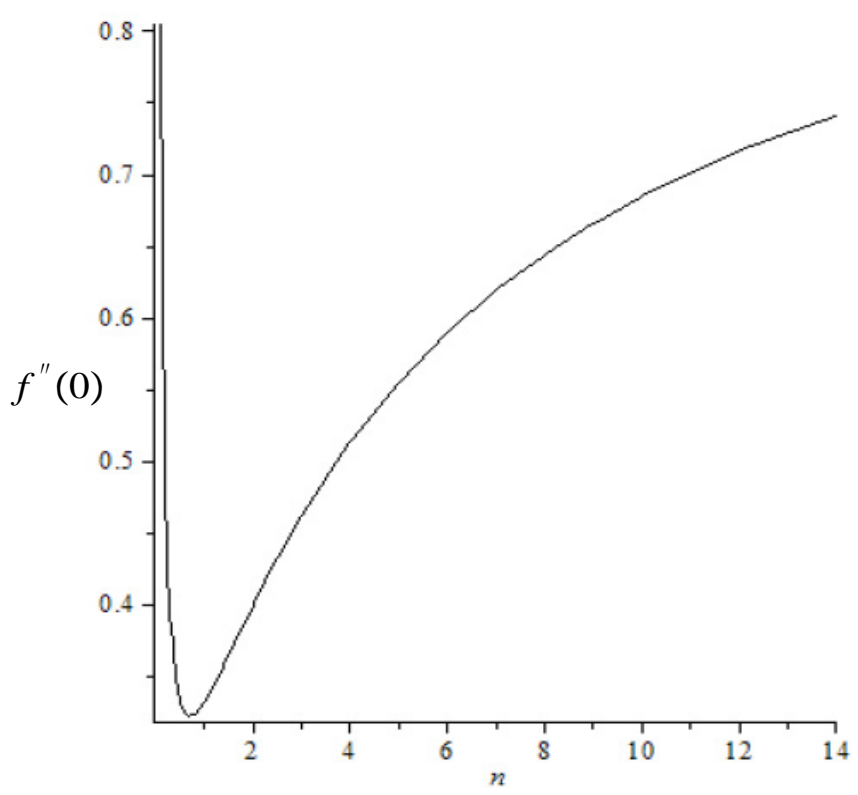

Figure 1. Velocity gradient parameter near the wall $f^{\prime \prime}(0)=\gamma$ against $n$ for $\lambda=0$

A Töpfer-like transformation is introduced by

$$
\begin{gathered}
g=\sigma^{\kappa} f, \\
\eta^{*}=\sigma^{\mu} \eta
\end{gathered}
$$

to convert the boundary value problem to an initial value problem. Equation (5) is scaling invariant if

$$
(2-n) \kappa=(1-2 n) \mu
$$




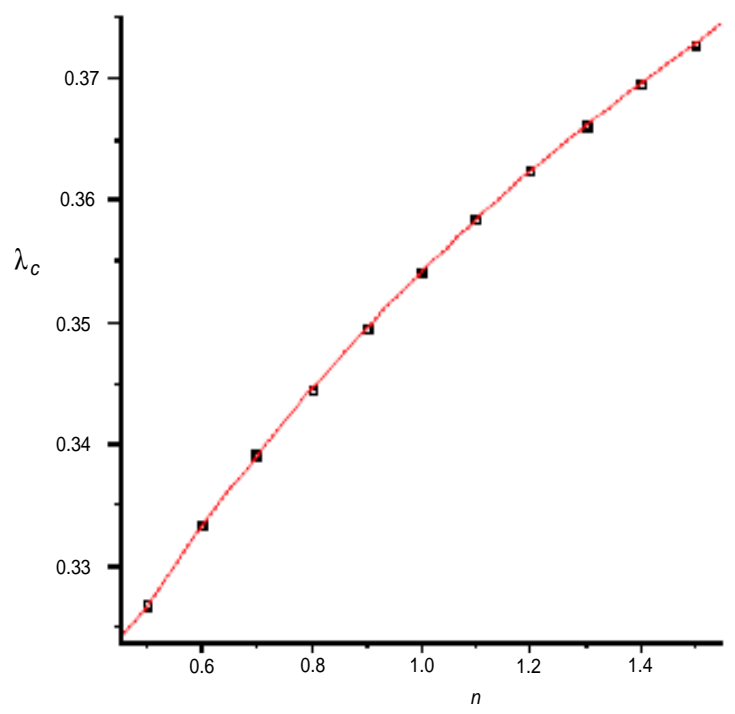

Figure 2. Critical velocity ratio $\lambda_{c}$ against $n$

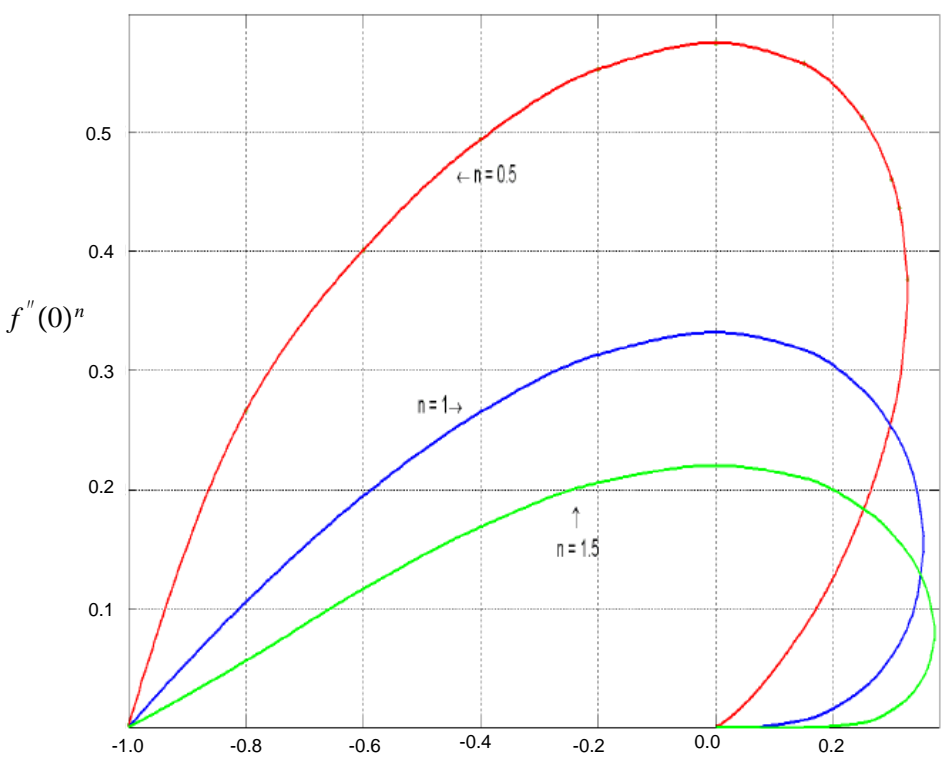

Figure 3. Drag coefficient parameter $f^{\prime \prime}(0)^{n}$ against $\lambda$ for different values of $n$ 
Then, one gets

$$
\left(\left|g^{\prime \prime}\right|^{n-1} g^{\prime \prime}\right)^{\prime}+\frac{1}{n+1} g g^{\prime \prime}=0
$$

Let us choose $\sigma=\gamma$, then

$$
g^{\prime \prime}(0)=\gamma^{\kappa-2 \mu+1}
$$

and with $\kappa-2 \mu+1=0$ one can obtain the appropriate boundary conditions as $\kappa=(1-2 n) / 3, \mu=(2-n) / 3$ :

$$
g(0)=0, \quad g^{\prime}(0)=-\lambda h^{*}, \quad g^{\prime \prime}(0)=1,
$$

where

$$
h^{*}=\gamma^{-\frac{n+1}{3}} h
$$

The initial value problem (4.6), 4.8) is solved with the so-called iterative transformation method. A numerical parameter $h$ is applied so that the asymptotic boundary condition remains invariant. By starting with a suitable value of $h^{*}$, a root finder algorithm is used to define a sequence $h_{i}^{*}$ for $j=0,1, \ldots$. The group parameter $\sigma$ is obtained by solving numerically the initial value problem after the iterations. The sequence is defined by

$$
\Gamma\left(h^{*}\right)=h-1=0 .
$$

An adequate termination criteria must be used to verify whether

$$
\Gamma\left(h_{i}^{*}\right) \rightarrow 0
$$

as $i \rightarrow \infty$. The solution of the original problem can be received by rescaling to $h=1$.

It is important to note that similarity solution exists only for $-1 \leq \lambda \leq \lambda_{c}$. If $\lambda>\lambda_{c}$, then the flow separates, the boundary layer structure collapses and the boundary layer approximations are no longer applicable.

Figure 2 provides an upper bound for the critical velocity parameter for nonNewtonian fluids.

The influences of $\lambda$ and $n$ on the skin friction parameter $\gamma$ are represented in Figure 3. It can be noticed that there are two solutions for $0<\lambda<\lambda_{c}$ (see Figure 3). 


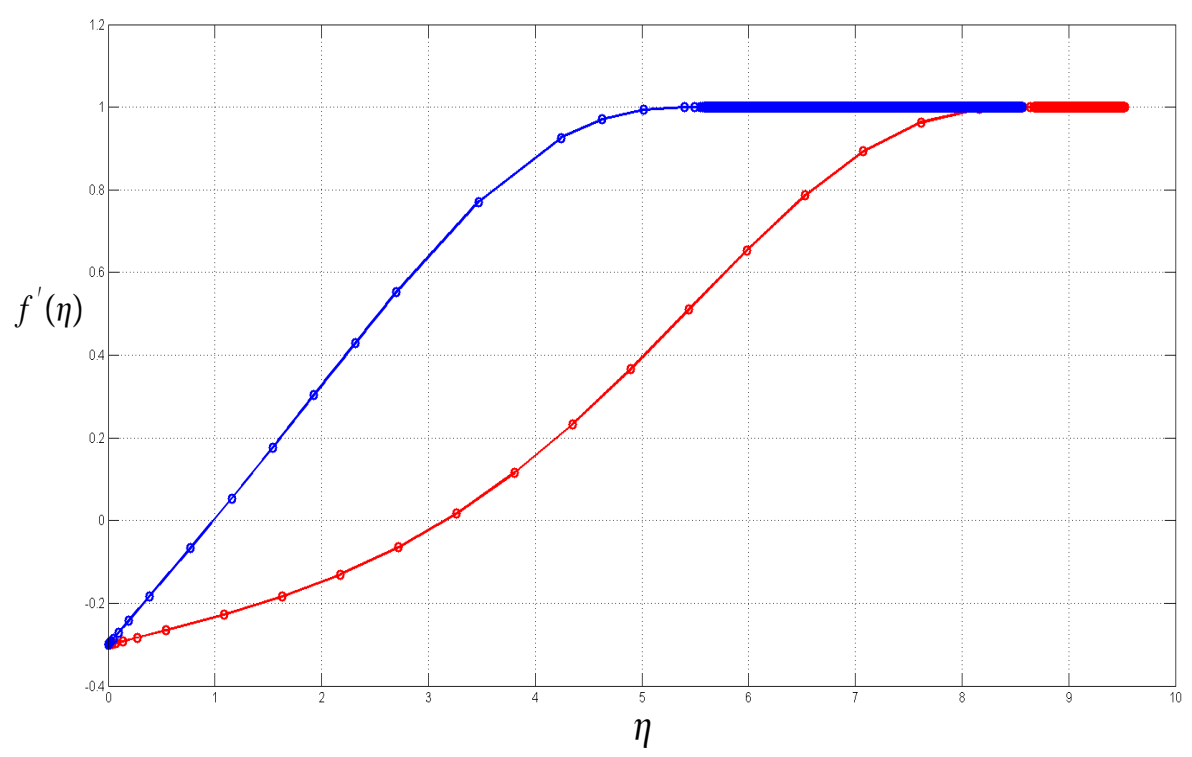

Figure 4. Velocity distribution for $n=1.5$ and $\lambda=0.3$

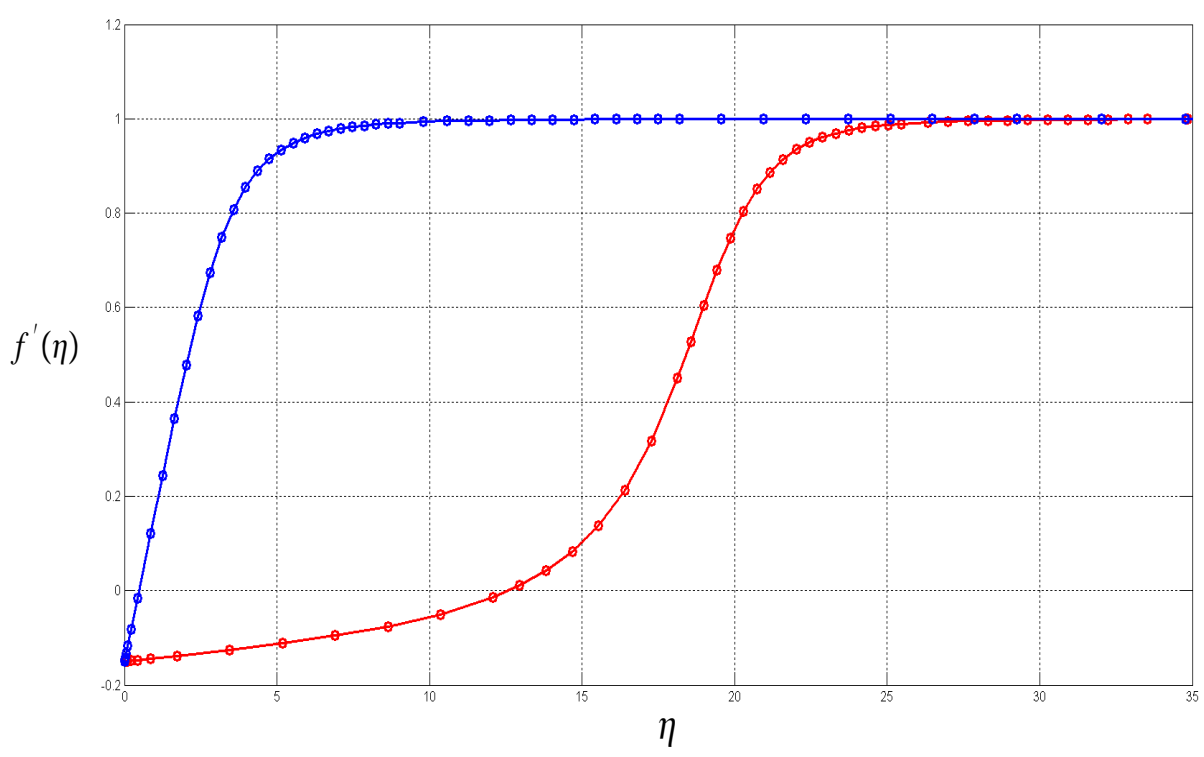

Figure 5. Velocity distribution for $n=0.5$ and $\lambda=0.15$ 
Figures 4.5 show the influence of the positive parameter $\lambda$ for different power-law exponent $n$ on $\gamma^{n}$. It can be seen that $f^{\prime}$ monotonically increases from $-\lambda$ to 1 for both the lower and upper solutions. These results are in good agreement with those reported in 23. Figures 46 exhibit the upper and lower solutions for velocities $f^{\prime}=u(x, y) / U_{\infty}$ as a function of $\eta$ for some values of $n$ and $\lambda$ to show the effect of the velocity parameter $\lambda$ and power-law exponent $n$. The figures show that $f^{\prime}$ monotonically increases from $-\lambda$ to 1 for both the lower and upper solutions. This phenomenon shows that the velocity component $u$ is monotonically increasing in the boundary layer.

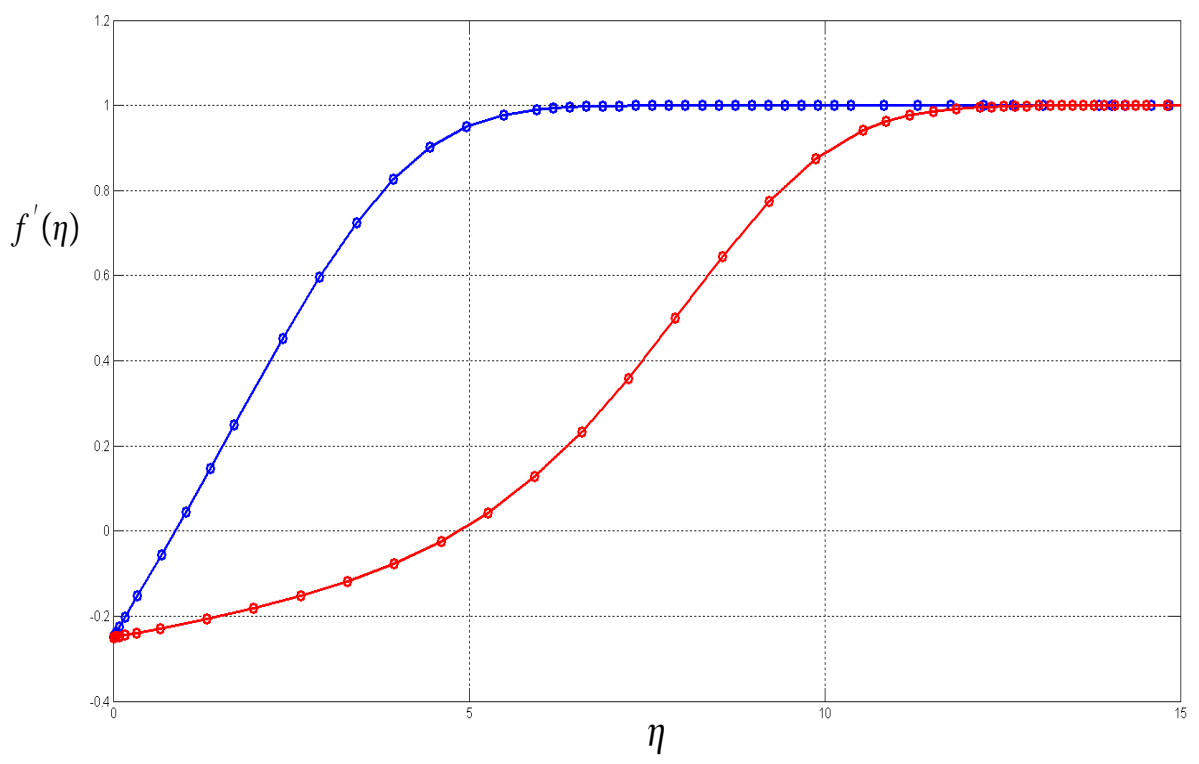

Figure 6. Velocity distribution for $n=1$ and $\lambda=0.25$

4.2. Spectral method for the case $U_{\infty}=0$. In this section a different method is introduced for the numerical solution to 2.10, 2.18 as the iterative transformation method applied in Section 4.1 is not suitable for this case.

With the advent of the spectral element method, complicated domains can be handled. In spite of being mainly used in fluid mechanics, nowadays, this method is more and more frequently utilized in biomechanics, astrophysics and in the study of electromagnetic waves.

We use a spectral method for the determination of the solution to 2.10, , 2.18). Spectral methods are able to provide very accurate results when the solution is smooth enough. More precisely, if the solution is differentiable to all orders, an exponential (or infinite order or spectral) convergence is achieved. 
All three versions of spectral methods (collocation, Galerkin and tau) belong to the method of weighted residuals and the main classification is carried out according to the type of trial functions used. Trial functions in the Galerkin method are the same as the weight functions and satisfy some of the boundary conditions. In spectral collocation, the trial functions are Dirac-delta functions located at the collocation points while the tau method, similarly to the Galerkin method, operates in the weak form but the trial functions generally do not satisfy the boundary conditions. In our calculations, the collocation method is used. During collocation we determine the function values of the interpolating polynomial at the collocation points (nodal approximation), as opposed to the other two methods, which give results for the coefficients of the truncated approximating series (modal approximation). The $n$-th order Chebyshev polynomial of the first kind, $T_{n}(x)$ is defined on $[-1 ; 1]$ and can be expressed by the recursion

$$
\begin{aligned}
& T_{0}(x)=1, T_{1}(x)=x, \\
T_{n}(x)= & 2 x T_{n-1}(x)-T_{n-2}(x), \quad n>1 .
\end{aligned}
$$

The modal approximation of function $u(x)$ is calculated by $T_{n}(x)$. The nodal approximation of $u(x)$ can be evaluated in the Lagrange base. The spectral differentiation for Chebyshev polynomials can be carried out either by a matrix-vector product or by using the Fast Fourier Transform (FFT). We implement the matrix-vector multiplication method because of the relatively low number of collocation points. One of the methods for solving a boundary value problem on an infinite or semi-infinite interval is the so-called domain truncation. Performing the truncation and the linear mapping we have

$$
\eta \in[0, \infty) \rightarrow \xi \in[0, L] \rightarrow \zeta \in[0,1] \rightarrow \bar{x} \in[-1,1]
$$

Introducing

$$
\bar{f}(\bar{x})=f(\eta(\bar{x})),
$$

the boundary value problem $2.10,2.18$ is written as

$$
\begin{aligned}
& \frac{8}{L^{3}} \bar{f}^{\prime \prime \prime}-\frac{1}{n(n+1)}\left(\frac{4}{L^{2}}\right) \bar{f}\left|\bar{f}^{\prime \prime}\right|^{2-n}=0, \\
& \bar{f}(-1)=0, \quad \bar{f}^{\prime}(-1)=L / 2, \quad \bar{f}(1)=0 .
\end{aligned}
$$

After the discretization, $N+2$ number of algebraic equations are at our disposal. The differential equation approximated at the $N-1$ inner nodes and the three boundary conditions. However, the number of unknowns is only $N+1$, therefore the resulting system is overdetermined. One possible solution is to take an interpolant that already satisfies some of the boundary conditions. Let us seek function $k$ such that

$$
\bar{f}(\bar{x})=P(\bar{x}) k(\bar{x}), \quad P(\bar{x})=a \bar{x}^{2}+b \bar{x}+c .
$$




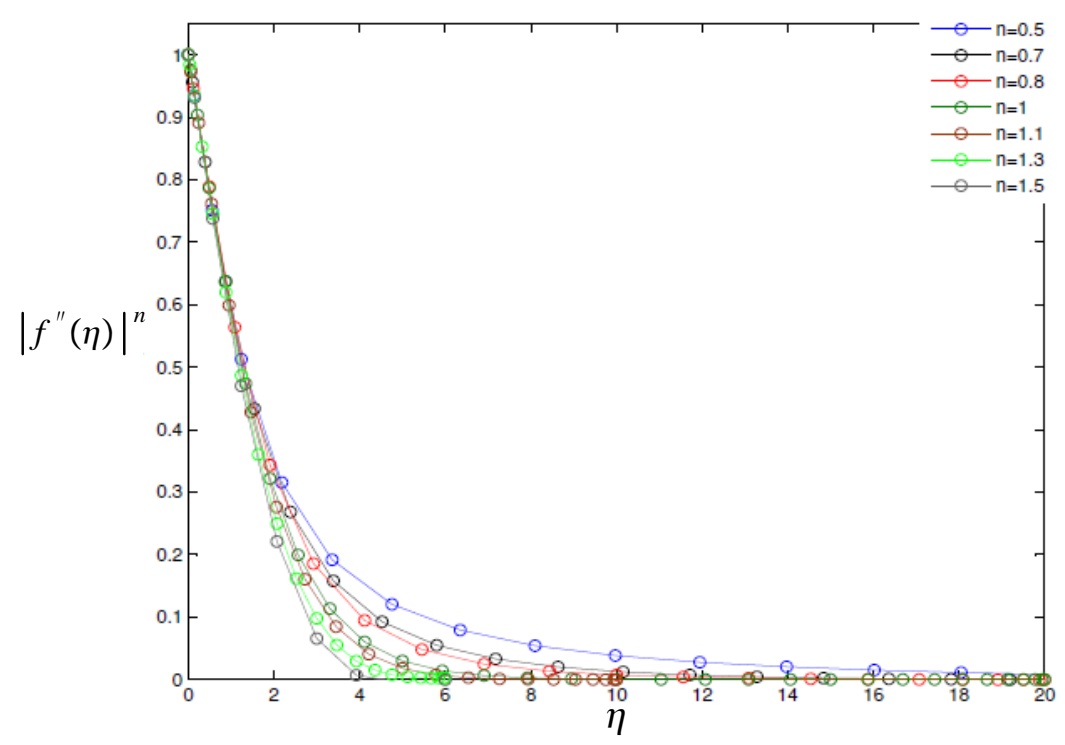

Figure 7. The values of $\left|f^{\prime \prime}(0)\right|^{n}$ for $n=0.5 \ldots 1.5$

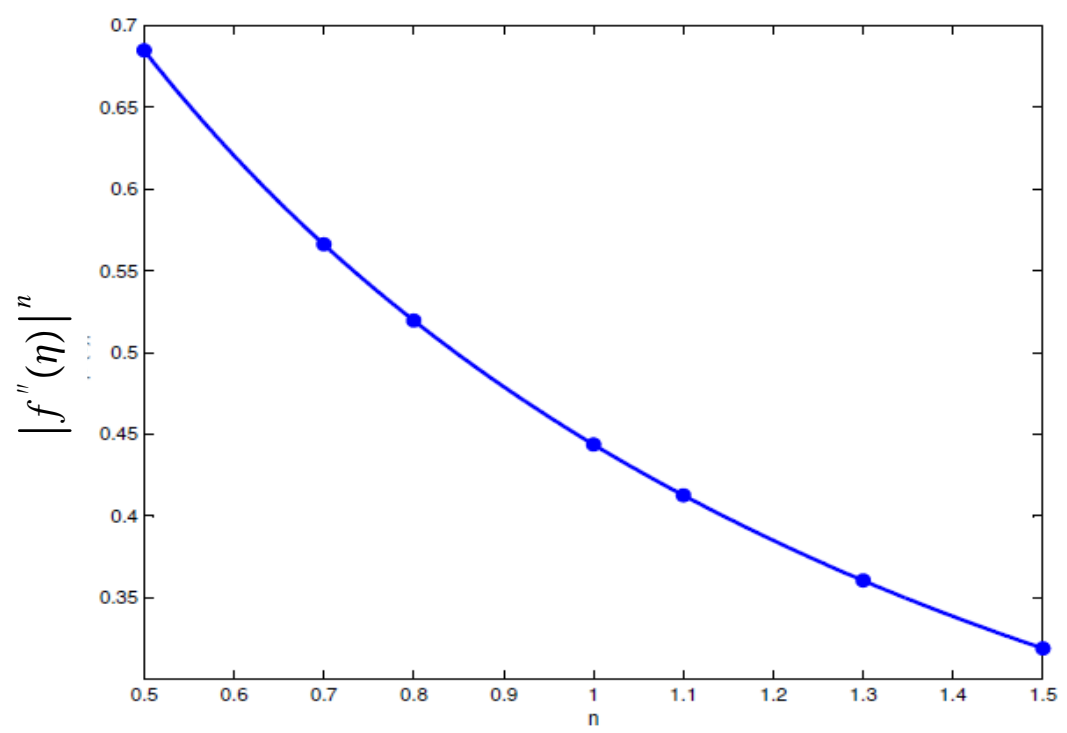

Figure 8. Velocity distribution for different values of $n$ for $U_{\infty}=0$

If conditions

$$
P(-1)=0, \quad P^{\prime}(-1)=L / 2
$$

and $P^{\prime}(1)=0$ are satisfied, then $a, b, c$ can be obtained as follows

$$
a=L / 8, \quad b=L / 4, \quad c=3 L / 8 .
$$


Now the differential equation is reformulated for $k$ under boundary conditions

$$
k(-1)=1, \quad k^{\prime}(1)=0 .
$$

The boundary value problem is solved with the Chebyshev spectral technique. After the discretization of $k(\bar{x})$ and its derivatives, the resulting system of nonlinear equations is solved with the Levenberg-Marquardt algorithm in Matlab. The numerical results for different power-law exponents are shown in Figure 7, where $f^{\prime}$ is shown, which is proportional to $u(x, y)$. The figure shows that with larger values of $n$ the boundary layer thickness decreases and shorter interval is enough for the truncation. Because $f^{\prime \prime}(0)<0$, both the drag coefficient and the wall shear stress are influenced by $\left|f^{\prime \prime}(0)\right|^{n}$ and this is exhibited in Figure 8 .

\section{Conclusions}

In this paper the determination of the drag coefficient is shown for two-dimensional, incompressible, laminar non-Newtonian fluid flow along a moving surface. The powerlaw non-Newtonian approximation is used. The governing partial differential equations are transformed into a third order ordinary differential equation together with the boundary conditions applying the similarity technique. Two main cases are considered: if the velocity $U_{\infty}$ of the ambient fluid flow is zero or non-zero. For $U_{\infty} \neq 0$, an iterative transformation method is used for the determination of the numerical results. If $U_{\infty}=0$, a spectral method is applied for the simulations. The values of $f^{\prime \prime}(0)=\gamma$ and the influence on it of the power-law exponent $n$ and the velocity ratio $\lambda$ on it are exhibited in Figures 18.

Acknowledgement. The research work presented in this paper is based on the results achieved within the TÁMOP-4.2.1.B-10/2/KONV-2010-0001 project and carried out as part of the TÁMOP-4.1.1.C-12/1/KONV-2012-0002 "Cooperation between higher education, research institutes and automotive industry project" in the framework of the New Széchenyi Plan. The realization of this project is supported by the Hungarian Government, by the European Union, and co-financed by the European Social Fund.

\section{REFERENCES}

1. Blasius, H.: Grenzschichten in Flussigkeiten mit kleiner reibung. Z. Math. Phys., 56, (1908), 1-37.

2. Schowalter, W. R.: The application of boundary layer theory to power-law pseudoplastic fluids: Similar solutions. AIChE J., 6, (1960), 24-28.

3. Acrivos, A., Shah M. J. and Peterson, E. E.: Momentum and heat transfer in laminar boundary flow of non-Newtonian fluids past external surfaces. AIChE J., 6, (1960), 312-317.

4. Sakiadis, B. C.: Boundary layers on continuous solid surfaces, AIChE. J., 7, (1961), 26-28, see also pp. 221-225 and 467-472.

5. Tsou, F., Sparrow, E. and Goldstein R.: Flow and heat transfer in the boundary layer on a continuous moving surface. Int. J. Heat Mass Transfer, 10, (1967), 219-235. 
6. Magyari, E. and Keller B.: Heat and mass transfer in the boundary layers on an exponentially stretching continuous surface. J. Phys. D: Appl. Phys., 32, (1999), 577585.

7. Magyari, E. and Keller B.: Exact solutions for self-similar boundary-layer flows induced by permeable stretching walls. Eur. J. Mech. B/Fluids, 19, (2000), 109-122.

8. LiaO S. J. and Pop, I.: Explicit analytic solution for similarity boundary layer equations. Int. J. Heat Mass Transfer, 47, (2004), 75-85.

9. Nazar R., Amin, N. and Pop, I.: Unsteady boundary layer flow due to a stretching surface in a rotating fluid, Mech. Res. Comm., 31, (2004), 121-128.

10. Shah Alam Md., Rashedul Islam Md., Ali M., Alim Md. A. and Alam Md. M.: Magnetohydrodynamic boundary layer flow of non-Newtonian fluid and combined heat and mass transfer about an inclined stretching sheet. Open J. App. Sci., 5, (2015), 279-294.

11. Wei D. M. and AL-Ashrab, S.: Similarity solutions for non-Newtonian power-law fluid flow. Appl. Math. Mech., 35, (2014), 1155-1166.

12. Grosan, T., Merkin, J. H. and Pop, I.: Mixed convection boundary-layer flow on a horizontal flat surface with a convective boundary condition. Meccanica, 48, (2013), 2149-2158.

13. Su X., Zheng, L. and JiAng, F.: Approximate analytical solutions and approximate value of skin friction coefficient for boundary layer of power law fluids, Appl. Math. Mech., 29, (2008), 1215-1220.

14. RAmA, B. and Goyal, M.: MHD non-Newtonian nano fluid flow over a permeable stretching sheet with heat generation and velocity slip. Int. J. Math. Comput. Nat. Phys. Eng., 8, (2014), 910-916.

15. Na Noghrehabadi A., Pourrajab R. and Ghalambaz M.: Effect of partial slip boundary condition on the flow and heat transfer of nano fluids past stretching sheet prescribed constant wall temperature. Int. J. Term. Sci., 54, (2012), 253-261. http: //dx.doi.org/10.1016/j.ijthermalsci.2011.11.017

16. Bognár, G.: Similarity solutions of boundary layer flow for non-Newtonian fluids. Int. J. Nonlinear Sci. Numer. Simul. 10, (2010), 1555-1566.

17. Benlahsen, M., Guedda, M. and Kersner, R.: The generalized Blasius equation revisited. Math. Comput. Model, 47, (2008), 1063-1076.

18. Weyl H.: On the differential equations of the simplest boundary-layer problems. Ann. of Math., 43, (1942), 381-407.

19. Hussaini M. Y. and LAKIN W. D.: Existence and nonuniqueness of similarity solutions of a boundary-layer problem, Q. J. Mech. Appl. Math., 39, (1986), 177-191.

20. Hussaini M. Y., Lakin W. D. and Nachman, A.: On similarity solutions of a boundary layer problem with an upstream moving wall, SIAMJ. Appl. Math., 47, (1987), 699-709.

21. Callegari, A. J. and Friedman, M. B.: An analytical solution of a nonlinear, singular boundary value problem in the theory of viscous flows. J. Math. Anal. Appl., 21, (1968), 510-529.

22. Nachman, A. and Callegari A. J.: A nonlinear singular boundary value problem in the theory of pseudoplastic fluids. SIAM J. Appl. Math., 38, (1980), 275-281. 
23. Ishak, A. and BaChok, N.: Power-law fluid flow on a moving wall, European J. Scie. Res., 34 (2009), 55-60.

24. BognÁR, G.: On similarity solutions of boundary layer problems with upstream moving wall in non-Newtonian power-law fluids. IMA J. Appl. Math., 77(4), (2012), 546-562.

25. FAzIO, R.: A novel approach to the numerical solution of boundary value problems on infinite intervals. SIAM J. Numer. Anal., 33, (1996), 1473-1483. 